\title{
Fisioterapia aquática em paciente com distrofia muscular de cinturas do tipo 2 b: relato de caso
}

\author{
Aquatic Physical therapy in a patient with Limb-Girdle \\ Muscular Dystrophy, type $\mathbf{2 b}$ : a case report
}

\author{
Layra Estelita Souza da Luz ${ }^{1}$ (1) \\ Rayssa de Cássia Ramos Nascimento 2 (1) \\ Mellina Monteiro Jacob ${ }^{3}$ (1) \\ Natáli Valim Oliver Bento-Torres ${ }^{4}$ (1)
}

\begin{abstract}
1-3Universidade Federal do Pará (Belém). Pará, Brasil. layraestelita@gmail.com, fisiorayssanascimento@gmail.com, mellinajacob@ufpa.br
\end{abstract} ${ }^{4}$ Autora para correspondência. Universidade Federal do Pará (Belém). Pará, Brasil. natalivalim@ufpa.br

RESUMO | INTRODUÇÃO: A distrofia muscular de cinturas do tipo 2B (DMC2B) é uma doença neuromuscular, degenerativa, rara, hereditária, progressiva, com consequentes prejuízos progressivos na capacidade motora e funcional. OBJETIVO: descrever e analisar os efeitos da fisioterapia aquática sobre a funcionalidade, força muscular, amplitude de movimento e qualidade de vida de uma paciente com diagnóstico DMC2B atendida em projeto de extensão universitária. MÉTODOS: Paciente do sexo feminino, 32 anos, solteira, com diagnóstico genético de Distrofia Muscular de Cinturas do Tipo 2B, nível 3 da escala Vignos (modificada por Garder-Medwin e Walton). O relato de caso apresenta a reabilitação através da Fisioterapia Aquática (hidrocinesioterapia) e seus impactos sobre a força muscular, amplitude de movimento, capacidade funcional e qualidade de vida da paciente (CAAE No. 43505321.0.0000.0018). RESULTADOS: O protocolo de fisioterapia aquática, composto por 12 sessões, $60 \mathrm{~min} / 2 \mathrm{x} / \mathrm{semana}$, resultou em melhoras na capacidade funcional global e aumento de 9,52\% na avaliação da função motora distal, aumentos de $100 \%$ da força de preensão manual e aumento para o limite superior (grau 5) na escala MRC para várias das musculaturas testadas, além de ganho de ADM e melhora expressiva da Qualidade de Vida. CONCLUSÃO: A melhora funcional apresentada pela paciente sugere que a reabilitação funcional fundamentada na fisioterapia aquática, em intensidade leve a moderada, é uma opção terapêutica segura e eficaz para a melhora da força muscular, amplitude de movimento, capacidade funcional e qualidade de vida na DMC2B.

PALAVRAS-CHAVE: Hidroterapia. Distrofia Muscular do Cíngulo dos Membros. Distrofias Musculares. Reabilitação. Fisioterapia. Relatos de Casos.
ABSTRACT | INTRODUCTION: Limb-Girdle Muscular Dystrophy, Type 2B (LGMD2B), is a rare, hereditary, progressive neuromuscular degenerative disease coursing with progressive impairments in motor and functional capacity. OBJECTIVE: To describe and analyze the effects of aquatic physical therapy on the functionality, muscle strength, range of motion, and quality of life of a patient diagnosed with LGMD2B attended on an outreach program. METHODS: A female patient, 32 years old, single, with genetic diagnosis of LGMD2B, level 5 at Vignos scale (modified by Garder-Medwin e Walton). The case reports the Aquatic Physical Therapy rehabilitation protocol (hydrokinesiotherapy) and its impacts on muscle strength, range of motion, functional capacity, and patient quality of life (CAAE No. 43505321.0.0000.0018). RESULTS: The aquatic physical therapy protocol, composed of 12 sessions, 60 minutes/2x/week, resulted in improvements in overall functional capacity and a $9.52 \%$ increase of distal motor function, $100 \%$ increase in handgrip strength, and increase up to the upper limit (grade 5) on the MRC scale for several of the muscles tested, in addition to increased range of motion and expressive improvement in Quality of Life. CONCLUSION: The patient' functional improvement suggests that water-based physical therapy rehabilitation, at mild to moderate exercise intensity, is a safe and effective therapeutic option for improvement muscle strength, range of motion, functional capacity, and quality of life in LGMD2B patients.

KEYWORDS: Hydrotherapy. Muscular Dystrophies, Limb-Girdle. Muscular Dystrophies. Rehabilitation. Physical Therapy Specialty. Case Reports. 


\section{Introdução}

As distrofias musculares de cinturas (DMC), são um grupo de doenças neuromusculares progressivas, hereditárias, raras e heterogêneas $\mathrm{s}^{1,2}$, com sintomas relacionados principalmente ao comprometimento dos músculos da cintura pélvica, escapular e de extremidade proximal ${ }^{3}$. Ocorrem diferenças da prevalência estimada para as DMC de acordo com os subtipos e populações específicas com prevalência de 1 a cada 14.000 a 45.000 indivíduos², sendo a distrofia muscular de cintura do tipo 2B (DMC2B) o segundo subtipo mais frequente em muitos países (15 a 25\%). Estudo brasileiro indicou prevalência de 9,79\% para DMC, sendo $18 \%$ correspondente a DMC2B. ${ }^{4}$

A patogênese da DMC2B é explicada por mutações autossômicas recessivas no gene DYSF, responsável pela codificação da proteína disferlina presente na membrana das células do músculo esquelético, tendo como consequência a fraqueza muscular, predominantemente da musculatura proximal ${ }^{5}$. Estudos recentes com ressonância magnética demonstram que músculos dos membros inferiores como glúteos, tensor da fáscia lata, semitendinoso, semimembranoso, bíceps femoral e tríceps sural são gravemente afetados. ${ }^{2}$

A amplitude de movimento articular, mobilidade, função muscular e qualidade de vida são comumente afetados com a progressão da doença, comprometendo a funcionalidade e causando prejuízos no contexto laboral, social e econômico ${ }^{6}$. Os comprometimentos cardíaco ou respiratório são incomuns e usualmente apresentam clínica silenciosa, podendo apresentar-se no final da vida. $\underline{\text { s }}$

Não existem terapias voltadas para a cura das DMC. As técnicas atuais de tratamento incluem terapia gênica e medicamentosa, intervenção cirúrgica, suporte para as funções cardíacas e respiratórias, suporte emocional e físico, além do uso de recursos auxiliares como bengalas, andadores e cadeira de rodas. A fisioterapia tem papel importante na manutenção e melhora da qualidade de vida dos pacientes portadores da DMC2B, objetivando o máximo de independência com ênfase em atividades funcionais e fortalecimento muscular. $\underline{9}$

Recente revisão sobre a intervenção através da fisioterapia aquática em pacientes com distrofia muscular aponta que não existem evidências de alto nível suficientes para suportarem a recomendação específica para a inclusão da fisioterapia aquática no tratamento de pacientes com distrofias musculares..$^{10}$ Achados da revisão relativos à Distrofia Muscular de Cinturas, objeto de interesse do presente manuscrito, são limitados a um único estudo de caso. ${ }^{11}$ É também importante registrar a escassez e necessidade do registro descritivos dos protocolos de intervenção em fisioterapia aquática, em associação a descrição do quadro funcional do paciente, para que a sua replicação na rotina clínica seja possível, justificando a importância do presente relato de caso, derivado de observações e intervenção realizadas na prática clínica..$^{10}$

Neste contexto, o objetivo do presente estudo é descrever e analisar os efeitos da fisioterapia aquática, associada a orientações para exercícios domiciliares em solo, sobre a funcionalidade, força muscular, amplitude de movimento e qualidade de vida de uma paciente com diagnóstico genético de DMC2B atendida em projeto de extensão universitária.

\section{Estudo de Caso}

\section{Apresentação do caso clínico}

Apresentamos o relato de caso do atendimento em projeto de ensino-extensão universitária de uma paciente do sexo feminino, 32 anos, solteira, destra, sedentária e sem histórico prévio de práticas esportivas ou de reabilitação funcional, com diagnóstico por exame genético de Distrofia Muscular de Cinturas do Tipo $2 \mathrm{~B}$, confirmado por laudo médico emitido por geneticista. A paciente foi classificada como nível 3 da escala Vignos (modificada por Garder-Medwin e Walton). ${ }^{12}$

A paciente, cujos pais são primos de primeiro grau, refere quatro familiares (um irmão, duas irmãs e um sobrinho) com confirmação diagnóstica por teste genético para a doença. Além de uma tia e uma prima com o mesmo quadro clínico e evoluções semelhantes. A equipe de pesquisa não teve acesso a informações clínicas e diagnósticas dos familiares.

Os sintomas iniciais da paciente manifestaram-se em 2008 (oito anos antes da participação no projeto de ensino-extensão), com falta de equilíbrio e fraqueza muscular em membros inferiores, porém, sem limitações de atividades. O quadro clínico evoluiu lentamente, progredindo, em 2011, para limitações nas 
atividades de subir escadas e ajoelhar, agravadas em 2014, a partir de quando passou a realizar essa atividade somente quando extremamente necessário, privilegiando o uso de rampas de acesso.

À avaliação cinético-funcional descritiva prévia à intervenção, em 2016, observou-se, de maneira geral, quadro de paraparesia de predomínio proximal, instabilidade postural, com queixas de dores articulares em quadril e joelhos, referindo cansaço aos médios esforços. A paciente realizava atividades de maneira independente, porém com limitações, tais como necessitar do auxílio de membros superiores para realizar mudanças na posição básica do corpo - e.g. trocas de decúbito, sentar e levantar ou transferir-se da posição ajoelhada para de pé. Ficava de pé de modo independente, necessitando de apoios eventuais em ponto fixo de MMSS, mantendo postura ereta com instabilidade, predominantemente utilizando base alargada em MMII. Apresentava padrão de marcha anserina e dificuldades - diminuição da velocidade e cansaço cardiorrespiratório - para caminhar médias distâncias. A paciente mantinha a funcionalidade na atividade de subir e descer escadas, necessitando de apoio de corrimão e maior tempo para o deslocamento.

As limitações de atividades acima descritas levaram à redução da participação da paciente em tarefas em grupo e inclusão social, especialmente aquelas associadas a tarefas de rotina do cargo religioso que ocupava, tais como transferir-se da posição ajoelhada para de pé e a limitações de deslocamento em conjunto com os demais pela diminuição da velocidade da marcha.

A paciente referiu, como queixa principal, dor na articulação do joelho esquerdo, cansaço aos médios esforços e limitação para as AVDs e direcionaram a elaboração do plano de tratamento. O presente estudo seguiu as recomendações da Resolução 466/2012 e Declaração de Helsinki e foi aprovado pelo Comitê de Ética Institucional (CAAE N 43505321.0.0000.0018). O Termo de Consentimento Livre e Esclarecido foi assinado pela paciente.

\section{Protocolo de Intervenção}

Foram realizadas 12 sessões (60min/2x/semana) de fisioterapia aquática, durante seis semanas, para o tratamento do quadro de redução de força muscular, dores nos joelhos e prejuízos na funcionalidade nas AVDs. Nos atendimentos foram realizados alongamentos, treinos funcionais, condicionamento cardiorrespiratório e fortalecimento muscular (Tabela 1), associados a orientação de exercícios domiciliares em solo para coordenação motora fina. A paciente não realizou nenhum outro tratamento físico concomitante à Fisioterapia Aquática.

Tabela 1. Protocolo de intervenção aquática

\begin{tabular}{|c|c|c|}
\hline Alongamento & $\begin{array}{c}\text { Treinamento Cardiorrespiratório } \\
\text { (treinos globais) }\end{array}$ & Fortalecimento Muscular* \\
\hline $\begin{array}{l}\text { 1. Extensores e flexores de ombro; } \\
\text { 2. Extensores e flexores de quadril; } \\
\text { 3. Extensores e flexores de joelho; } \\
\text { 4. Flexores plantar e dorsiflexores; } \\
\text { 5. Dissociação de cinturas em } \\
\text { flutuação*. }\end{array}$ & $\begin{array}{l}\text { 1. Conscientização do padrão } \\
\text { respiratório; } \\
\text { 2. Movimento de bicicleta em } \\
\text { flutuação*; } \\
\text { 3. Marcha para frente e para trás } \\
\text { (passos longos e turbulência } \\
\text { frontal); } \\
\text { 4. Movimentos de natação \# } \\
\text { (crawl, peito e costa). }\end{array}$ & $\begin{array}{l}\text { 1. Flexores, extensores, adutores e } \\
\text { abdutores de ombro; } \\
\text { 2. Flexores e extensores de cotovelo; } \\
\text { 3. Flexão e extensão de punho; } \\
\text { 4. Abdominal; } \\
\text { 5. Flexores, extensores, adutores e } \\
\text { abdutores de quadril; } \\
\text { 6. Flexores e extensores de joelho; } \\
\text { 7. Flexores plantar e dorsiflexores de } \\
\text { tornozelo. }\end{array}$ \\
\hline
\end{tabular}

${ }^{*}$ ) Foram utilizados macarrão, prancha, halter e palmar de acordo com a necessidade de sustentação ou resistência em cada movimento.

(\#) Os diversos movimentos de natação foram realizados de acordo com as potencialidades e as possibilidades da paciente em ambiente aquático. 
Alongamentos globais para grandes grupos musculares em membros superiores (MMSS) e membros inferiores (MMII) (evoluindo de estáticos para dinâmicos) ou alongamentos passivos em flutuação foram empregados no início e fim de cada sessão. O treinamento cardiorrespiratório foi realizado através de exercícios de caminhada contra a turbulência, padrões de natação ou movimentos simulados de pedalar em bicicleta em flutuação. Esta etapa apresentou duração média de 30 minutos e foi realizada em intensidade equivalente ao nível 5 da escala de Borg (intensidade leve-moderada). Os exercícios para fortalecimento muscular foram realizados contra a resistência da água, com o uso de recursos como pranchas, halteres, flutuadores e palmares, com duração média de 20 minutos. Associado à execução do exercício, as terapeutas executavam a estimulação manual (Tapping de deslizamento) como feedback tátil para potencializar a contração muscular.

A progressão do nível de dificuldade para impor maior resistência ao movimento foi realizada através da manipulação de variáveis: velocidade e direção de movimento; forma, posição ou profundidade dos equipamentos; uso de turbulência. A paciente foi também orientada a realizar exercícios de solo, com enfoque na coordenação motora fina de mãos e dedos, utilizando-se materiais de fácil acesso, tais como pedras pequenas e grãos culinários.

\section{Avaliações}

A avaliação fisioterapêutica foi realizada por único avaliador, antes do início (AV1) e após o final (AV2) do protocolo de intervenção. Foram avaliados aspectos relacionados à história clínica, capacidade funcional (escala Medida da Função Motora - MFM), força muscular (Escala Medical Research Council - MRC e dinamometria manual), amplitude de movimento (goniometria manual), e qualidade de vida (SF-36). Todos os testes são amplamente utilizados no meio científico e clínico para avaliação dos pacientes com doenças neuromusculares. $13-11$

\section{Capacidade Funcional}

A escala Medida da Função Motora (MFM) é específica e sensível para a avaliação funcional de pacientes portadores de doenças neuromusculares. ${ }^{18}$ Esta escala é composta por 32 itens, divididos em três dimensões (D1, D2 e D3), sendo desenhada para fornecer perfil detalhado da função motora da posição em pé e transferências (D1), função motora axial e proximal (D2) e função motora distal (D3). A pontuação total varia de 0 a 96 pontos, equivalentes a baixa e alta função motora, respectivamente, sendo também possível calcular o valor de cada dimensão em porcentagem. $\frac{15}{}$

\section{Força muscular}

A avaliação da força muscular foi realizada por meio da avaliação manual (escala MRC) e da medida da força de preensão (dinamometria). ${ }^{13,14,19} \mathrm{O}$ escore da MRC varia de ' 0 ' a ' 5 ', em que ' 0 ' representa ausência de resposta muscular visível, '1' contração muscular visível ou palpável, sem movimento do membro, '2' movimento do membro, mas não contra a gravidade e ' 3 ' Movimento contra a gravidade em quase toda a amplitude de movimento, '4' Movimento contra resistência moderada em toda a amplitude de movimento e '5' Movimento Normal. A força muscular foi avaliada bilateralmente em: bíceps braquial, tríceps braquial, deltóides, flexores de punho, extensores de punho, adutores de ombro, quadríceps femoral, iliopsoas, isquiotibiais, abdutores de quadril, gastrocnêmios, adutores de quadril, tibial anterior, glúteos médio e máximo.

A força de preensão manual foi avaliada com uso do dinamômetro hidráulico (Jamar $\left.{ }^{\circledR}\right)$, seguindo as recomendações de posicionamento e coleta dos dados da American Society of Hand Therapists. 19 Três tentativas foram realizadas, com intervalos de 30 segundos entre aferições, e o melhor desempenho foi utilizado na análise.

\section{Amplitude de movimento (ADM)}

A avaliação da ADM foi realizada por goniometria manual (goniômetro Carci ${ }^{\circledR}$ ), método de utilização universal, com vantagens que incluem baixo custo do instrumento e facilidade de medição, sendo importante na verificação de intervenções terapêuticas. A ADM foi avaliada ao final da amplitude total de movimento, bilateralmente, em flexão, extensão, abdução e adução de ombros e quadril, flexão e extensão de cotovelos, punho, joelhos, assim como flexão-plantar e dorsiflexão de tornozelos. 


\section{Qualidade de vida}

A qualidade de vida foi avaliada através da aplicação do questionário SF-36 (Short Form Health Survey 36), instrumento composto por 36 itens, amplamente usado para avaliação da qualidade de vida relacionada à saúde, inclusive na população com distrofias musculares. $\frac{16,17}{}$ O SF-36 avalia oito domínios da saúde: funcionamento físico, limitações de função devido a problemas de saúde física, dor corporal, saúde geral, vitalidade, funcionamento social, limitação de função devido a problemas emocionais e saúde mental. A pontuação total é de 0 (zero) à 100 (cem) pontos, sendo que pontuações mais altas indicam melhor estado geral de saúde.

\section{Resultados}

Durante a avaliação inicial a paciente apresentava-se em bom estado geral de saúde. Foi observada marcha independente, padrão anserino e fraqueza em membros superiores (MMSS) e membros inferiores (MMII).

$\mathrm{Na}$ avaliação pela escala MFM, após as 12 sessões de fisioterapia aquática, foi observado aumento de $6,25 \%$ na pontuação total da capacidade funcional, havendo melhora de 7,69\% da função motora da posição em pé e transferências, 2,78\% na função motora axial e proximal e 9,52\% na função motora distal, sendo esta última a dimensão que demonstrou maior ganho percentual (Tabela 2).

Tabela 2. Capacidade Funcional. Pontuações em cada dimensão nas avaliações pela Escala Medida da Função Motora (MFM) pré (AV1) e pós (AV2) intervenção, assim como a melhora percentual em cada dimensão

\begin{tabular}{cccc}
\hline $\begin{array}{c}\text { Dimensões da escala } \\
\text { MFM }\end{array}$ & AV1 (\%) & AV2 (\%) & Melhora (\%) \\
\hline D1 & 66,7 & 74,36 & 7,69 \\
D2 & 97,22 & 100,00 & 2,78 \\
D3 & 85,71 & 95,24 & 9,52 \\
\hline Total & 82,29 & 88,54 & 6,25 \\
\hline
\end{tabular}

AV1: avaliação inicial; AV2: avaliação final; \%: percentual. D1: Dimensão 1 - Função motora da posição em pé e transferências; D2: Dimensão 2 - Função motora axial e proximal; D3: Dimensão 3 - Função motora distal.

A avaliação inicial da força muscular pela escala MRC indicou prejuízo tanto na musculatura distal quanto proximal, com maiores comprometimentos da força muscular de membros inferiores (MRC scores $\leq 4$ ) do que superiores (MRC scores $\geq 4$ ) (Tabela 3). Após tratamento, avaliou-se aumento de 1 grau de força em flexores de punho, extensores de punho, adutores de ombro, bíceps braquial e deltóide, assim como manutenção da força aferida em tríceps braquial, finalizando a intervenção com força máxima (grau 5) em membros superiores para todos os grupos musculares testados como grau 4, à avaliação inicial (Tabela 3 ).

Em MMII houve manutenção da força do quadríceps femoral seguida de aumento de 1 grau de força para os músculos gastrocnêmio, tibial anterior, glúteo médio, glúteo máximo, isquiotibiais, adutores de quadril e iliopsoas. A avaliação dos músculos abdutores de quadril indicou aumento de 2 graus de força, finalizando a intervenção com grau 4 nesse grupamento muscular, (Tabela 3). 
Tabela 3. Avaliação da força muscular pela escala Medical Research Council (MRC). Resultados apresentados em valores absolutos para as avaliações pré (AV1) e pós (AV2) intervenção

\begin{tabular}{|c|c|c|c|c|}
\hline \multirow[t]{2}{*}{ Musculatura avaliada } & \multicolumn{2}{|c|}{ Membro Superior Direito } & \multicolumn{2}{|c|}{ Membro Superior Esquerdo } \\
\hline & AV1 & AV2 & AV1 & AV2 \\
\hline Bíceps Braquial & 5 & 5 & 4 & 5 \\
\hline Tríceps Braquial & 5 & 5 & 5 & 5 \\
\hline Deltóide & 5 & 5 & 4 & 5 \\
\hline Flexores de Punho & 4 & 5 & 4 & 5 \\
\hline Extensores de Punho & 4 & 5 & 4 & 5 \\
\hline Adutores de Ombro & 4 & 5 & 4 & 5 \\
\hline \multirow[t]{2}{*}{ Musculatura avaliada } & \multicolumn{2}{|c|}{ Membro Inferior Direito } & \multicolumn{2}{|c|}{ Membro Inferior Esquerdo } \\
\hline & AV1 & AV2 & AV1 & AV2 \\
\hline Quadríceps Femoral & 4 & 4 & 4 & 4 \\
\hline íliopsoas & 4 & 5 & 4 & 5 \\
\hline Isquiotibial & 3 & 4 & 3 & 4 \\
\hline Abdutores de Quadril & 2 & 4 & 2 & 4 \\
\hline Gastrocnêmio & 1 & 2 & 1 & 2 \\
\hline Adutores de Quadril & 4 & 5 & 4 & 5 \\
\hline Tibial Anterior & 1 & 2 & 1 & 1 \\
\hline Glúteo Máximo & 4 & 5 & 4 & 4 \\
\hline Glúteo Médio & 1 & 2 & 1 & 2 \\
\hline
\end{tabular}

AV1: avaliação inicial; AV2: avaliação final.

A avaliação da preensão manual indicou aumento de 53,3\% e 100\% da força manual após a intervenção para membro superior direito e esquerdo, respectivamente (MSD - AV1: 7,5 KgF, AV2: 11,5 Kg; MSE - AV1: 5 KgF, AV2: $10 \mathrm{KgF}$ ). Foi realizada avaliação da amplitude de movimento (ADM) das principais articulações de membros superiores e inferiores, registrando-se aumento da angulação medida em todas as articulações testadas após o protocolo de intervenção aquática. É importante destacar que, na avaliação inicial, não foram registradas limitações mecânicas ou rigidez articular aos movimentos.

Os maiores ganhos de amplitudes articular de movimento foram observados na flexão do ombro (MSD: +42 ;

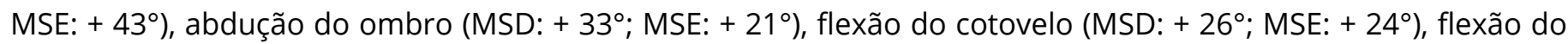
joelho (MID: $+13^{\circ} ;$ MIE: $+7^{\circ}$ ), extensão do joelho (MID: $+10^{\circ} ;$ MIE: $\left.+19^{\circ}\right)$, abdução do quadril (MID: + $10^{\circ} ;$ MIE: + $6^{\circ}$ ), além de flexão plantar na articulação do tornozelo (MID: $1,0^{\circ}$; MIE: $4^{\circ}$ ).

A percepção subjetiva da qualidade de vida, avaliada pelo SF-36, indicou melhora após a intervenção pela fisioterapia aquática. Os domínios em que a paciente apresentou maior ganho foram aqueles relacionados aos aspectos físicos, dor e capacidade funcional, com melhora de $400 \%, 80 \%$ e 37,5\%, respectivamente. Nos outros domínios analisados houve manutenção ou aumento do escore após a reabilitação, exceto no domínio saúde mental, conforme apresentado na Tabela 4. 
Tabela 4. Avaliação da Qualidade de Vida Percebida (SF-36). Valores em pontos obtidos na AV1 e AV2 e em percentual após a intervenção

\begin{tabular}{lccc}
\hline \multicolumn{1}{c}{ Domínios } & AV1 & AV2 & Valor (\%) \\
\hline Capacidade funcional & 40 & 55 & $+37,5$ \\
Limitações por aspectos físicos & 25 & 100 & +400 \\
Estado geral de saúde & 40 & 47 & $+17,5$ \\
Vitalidade & 65 & 75 & $+15,38$ \\
Limitações por aspectos emocionais & 100 & 100 & 0 \\
Dor & 40 & 72 & +80 \\
Saúde mental & 88 & 72 & $-18,18$ \\
Aspectos sociais & 100 & 100 & 0 \\
\hline AV1: avaliação inicial: AV2: avaliação final; \%: porcentagem; +*ganho; -: perda. &
\end{tabular}

\section{Discussão}

O presente estudo teve como objetivo analisar o efeito de um protocolo de fisioterapia aquática sobre a funcionalidade, força muscular, amplitude de movimento e qualidade de vida de uma paciente portadora da Distrofia Muscular de Cinturas do Tipo 2B (DMC2B). Nossos resultados demonstram que o protocolo aplicado em 12 sessões de fisioterapia aquática resultou em melhoras na capacidade funcional global e aumento de 9,52\% na avaliação da função motora distal, aumentos de $100 \%$ da força de preensão manual de MSE e aumento para o limite superior (grau 5) na escala MRC para todas as musculaturas que à avaliação apresentaram grau 4, além de ganho de ADM e melhora expressiva da Qualidade de Vida.

É recomendação internacional a adoção de estilo de vida fisicamente ativo e a prática de exercícios físicos para a promoção da saúde, prevenção e tratamento de diferentes doenças. Todavia, a recomendação pela intervenção por exercícios físicos é controversa em pessoas com doenças neuromusculares, apesar de existirem evidências dos seus benefícios para o tratamento desse grupo de pacientes. ${ }^{14,20,21}$

Por tratar-se de doença rara, resultante de mutação genética recessiva específica, não estão disponíveis estudos que investiguem estratégias de reabilitação funcional específicas para as pessoas acometidas pela Distrofia Muscular de Cinturas do Tipo 2B (DMC2B). 10 Nesse grupo específico de pacientes, o gene DYSF - responsável pela codificação da proteína disferlina - é deficiente ou ausente, levando a prejuízos da manutenção da estrutura da fibra muscular, ocasionando rompimento do sarcolema e desestruturação da unidade motora, com consequente perda de força muscular e função motora. Os danos musculares durante os exercícios também têm sido associados, de modo inconclusivo, a alterações da excitação-contração muscular, do metabolismo oxidativo e/ou energético. 21

Essa característica fisiopatológica pode impor limites à tolerância ao exercício físico por conta da perda de fibras musculares ou pela diminuição do nível de atividade física associado ao comprometimento motor. $\stackrel{21}{\text { Devido a }}$ condição de degeneração muscular nas distrofias, a tolerância é especificamente diminuída aos exercícios físicos de alta intensidade e contrações excêntricas, os quais podem induzir dano muscular e subsequente fraqueza do músculo. Em contraponto, há efeito positivo do exercício físico em diversas doenças neuromusculares, sem associação à suscetibilidade à lesão muscular, prevenindo assim a atrofia por desuso, desde que praticados em intensidades leve a moderada, dentro dos limites de tolerância do paciente. .1, $22^{2}$ O protocolo do presente estudo foi realizado em intensidade leve a moderada e gerou melhoras físicas e funcionais na paciente, sem registros de intercorrências ou sinais de sobrecarga em quaisquer das sessões ou pós-treino. 
Nesse contexto, a fisioterapia aquática pode ser uma alternativa terapêutica eficaz para o tratamento dos pacientes portadores de DMC2B pela possibilidade de tratar os prejuízos impostos pela distrofia em um ambiente de maior suporte e controle associados aos princípios fundamentais da hidrodinâmica, como densidade, arrasto, flutuabilidade, pressão hidrostática e termodinâmica. Dentre eles, a flutuabilidade destaca-se, pois pode reduzir a sobrecarga em até $85 \%$ nas articulações submersas, permitindo assim maior fluidez no manejo do paciente e menor sobrecarga articular. $\frac{23}{}$ Apesar dos potenciais benefícios e vantagens da reabilitação aquática, até o presente momento, não foram localizados artigos que a reportem como estratégia terapêutica direcionada ao tratamento das alterações de pacientes portadores da DMC2B.

Com base na classificação da corte de Harris e colaboradores ${ }^{5}$, a paciente avaliada no presente estudo apresenta comprometimento moderado, apresentando os padrões de comprometimento muscular descritos para as distrofias musculares de cinturas. Apresenta como característica clínica a fraqueza mais severa dos músculos de membros inferiores do que superiores e, dentre aqueles, os músculos proximais da cintura pélvica, das coxas e flexores do tornozelo, levando ao predomínio de dificuldades dependentes do recrutamento desses músculos, tais como levantar de uma cadeira ou subir escadas..$^{13}$ No entanto, o comprometimento de força muscular em membros superiores manifesta-se com um padrão diferente do padrão típico da maioria da população acometida ${ }^{22}$, com assimetria entre a força muscular do músculo deltoide e bíceps braquial direitos e esquerdos, mantendo, todavia simetria para as demais musculaturas.

Estudo com ressonância magnética aponta a substituição de massa muscular por tecido adiposo em $88 \%$ dos pacientes estudados, sendo maior o comprometimento da região posterior das coxas e pernas. ${ }^{24} \mathrm{O}$ treino com ênfase na musculatura mencionada foi realizado, valorizando-se as adaptações e ajustes de movimentos dos principais tipos de nado, dentro das possibilidades funcionais da paciente em ambiente aquático, realizando os movimentos mesmo que executados fora dos padrões de técnica.

Melhoras funcionais foram encontradas na avaliação da força muscular, funcionalidade e qualidade de vida da paciente atendida no projeto de extensão.
Destaca-se que a paciente não apresentava histórico prévio de prática de exercícios físicos, esportes ou quaisquer tratamentos físicos/fisioterapêuticos, além de ter hábitos de vida sedentária, o que gera maior lastro potencial para os benefícios advindos do exercício físico, mesmo em curto período de intervenção. Esses resultados estão alinhados com resultados anteriores que sugerem que um programa de treinamento de força supervisionado, associado com exercícios aeróbios de baixo impacto, como natação e bicicleta estacionária, melhoram o desempenho cardiovascular, eficácia muscular e diminuição da fadiga de pacientes portadores de distrofia muscular de cinturas ${ }^{22}$, descrevem intervenções por fisioterapia aquática de mesma duração e periodicidade em pacientes com distrofias ${ }^{10}$ e recomendam o exercício físico e o combate à inatividade física como parte do tratamento. 14

Estudo recentemente publicado ${ }^{18}$ avaliou a sensibilidade de instrumentos e medidas para avaliação das alterações associadas à progressão da patologia, indicando que a pontuação total da escala MFM é um dos instrumentos mais sensíveis para avaliação e monitoramento das alterações clínicas da função motora, demonstrando alterações consistentes ao longo do tempo, especialmente em pacientes DMC2B.

Considerando a progressão lenta característica da DMC2B, estabelecer estratégias de tratamento e reabilitação funcional é importante para a manutenção da independência funcional pelo maior tempo possível. A falta de ensaios clínicos e estudos de alto nível de evidência limitam o estabelecimento de protocolos de intervenção ou modalidade terapêutica com eficácia avaliada para o tratamento e reabilitação dos pacientes portadores de DMC2B e agregam valor a evidências de tratamentos fisioterapêuticos bem-sucedidos, como o apresentado no presente relato de caso. Todavia, as evidências disponíveis hoje apontam que os exercícios físicos terapêuticos devem fazer parte da rotina da reabilitação dos pacientes com distrofias musculares, o que foi corroborado pelos achados descritos no presente estudo apontando a fisioterapia aquática como segura e eficaz no tratamento de uma paciente portadora de distrofia muscular de cinturas do tipo 2B.

Sendo assim novos estudos com maior número de participantes são necessários para produzir evidências que permitam a generalização dos dados quanto 
à eficácia da Fisioterapia Aquática no escopo de cuidados aos pacientes portadores da Distrofia Muscular de Cinturas do Tipo 2B (DMC2B).

\section{Conclusão}

A ausência de estratégias terapêuticas para o controle da progressão da DMC2B aumenta a importância dos tratamentos fisioterapêuticos com vistas à reabilitação funcional, à manutenção da funcionalidade dos pacientes e aumento da atividade física nessa população. A melhora funcional apresentada pela paciente atendida no projeto de extensão sugere que a reabilitação funcional fundamentada na Fisioterapia aquática, em intensidade leve a moderada, foi uma opção terapêutica segura e eficaz para o ganho da força muscular, amplitude de movimento, capacidade funcional e qualidade de vida das disfunções motoras e funcionais associadas ao caso descrito.

\section{Agradecimentos}

As autoras agradecem a Sra. Krinten Novak pela revisão gramatical completa da versão em inglês do manuscrito.

\section{Contribuições dos autores}

Bento-Torres NVO, Luz LES e Nascimento RCR participaram da concepção do estudo, do atendimento a paciente, análise dos dados, interpretação dos resultados e escrita do manuscrito. Jacob MM contribuiu para a análise dos dados e escrita do manuscrito. Todos os autores leram e aprovaram a versão final do manuscrito.

\section{Conflitos de interesses}

Nenhum conflito financeiro, legal ou político envolvendo terceiros (governo, empresas e fundações privadas, etc.) foi declarado para nenhum aspecto do trabalho submetido (incluindo, mas não se limitando a subvenções e financiamentos, participação em conselho consultivo, desenho de estudo, preparação de manuscrito, análise estatística, etc.).

\section{Referências}

1. Cotta A, Carvalho E, da-Cunha-Júnior AL, Paim JF, Navarro MM, Valicek J, et al. Diagnóstico diferencial das distrofias musculares cintura-membros recessivas comuns: como e por quê? Arq Neuro-Psiquiatr. 2014;72(9):721-34. https://doi.org/10.1590/0004$\underline{282 \times 20140110}$
2. Murphy AP, Straub V. The Classification, Natural History and Treatment of the Limb Girdle Muscular Dystrophies. J Neuromuscul Dis. 2015;2(s2):S7-19. https://doi.org/10.3233/jnd150105

3. Wicklund MP, Kissel JT. The limb-girdle muscular dystrophies. Neurol Clin [Internet]. 2014;32(3):729-49. Disponível em: https:// aica3.org/cms/wp-content/uploads/2015/02/LGMDrev2014.pdf

4. Cotta A, Paim JF, Carvalho E, da-Cunha-Júnior AL, Navarro $M M$, Valicek J, et al. Frequência relativa de diagnósticos neuromusculares comuns em um serviço de referência. Arquivos de neuro-psiquiatria. 2017;75(11):789-95. https://doi. org/10.1590/0004-282X20170151

5. Harris E, Bladen CL, Mayhew A, James M, Bettinson K, Moore $U$, et al. The Clinical Outcome Study for dysferlinopathy: An international multicenter study. Neurol Genet. 2016;2(4):e89. https://doi.org/10.1212/nxg.0000000000000089

6. Aimee K, Kathleen M. Aquatic and Land-Based Physical Therapy Improves Functional Mobility and Quality of Life in a Patient with Bethlem Myopathy Muscular Dystrophy: A Case Report. Physiother Rehabil. 2017;2(1):133. http://dx.doi.org/10.4172/2573$\underline{0312.1000133}$

7. Schneider I, Stoltenburg G, Deschauer M, Winterholler M, Hanisch F. Limb girdle muscular dystrophy type $2 \mathrm{~L}$ presenting as necrotizing myopathy. Acta Myol. 2014;33(1):19-21. Citado em: PMID: 24843231

8. Mahmood OA, Jiang XM. Limb-girdle muscular dystrophies: where next after six decades from the first proposal (Review). Mol Med Rep. 2014;9(5):1515-32. https://doi.org/10.3892/ $\underline{\mathrm{mmr} .2014 .2048}$

9. Kilinç M, Yildirim SA, Tan E. The effects of electrical stimulation and exercise therapy in patients with limb girdle muscular dystrophy. A controlled clinical trial. Neurosciences (Riyadh). 2015;20(3):259-66. https://dx.doi. org/10.17712\%2Fnsj.2015.3.201501097

10. Lima AA, Cordeiro L. Fisioterapia aquática em indivíduos com distrofia muscular: uma revisão sistemática do tipo escopo. Fisioter Pesqui. 2020;27(1):100-11. https://doi.org/10.1590/18092950/18031327012020

11. DiBiasio P, Primus KB, Drogos J, Husted C, Stephens H, Thorpe $D$. An aquatic exercise program for an adolescent with limb girdle muscular dystrophy. J Aquat Phys Ther [Internet]. 2015;23(1):3-14. Disponível em: https://journals.Iww.com/japt/ Abstract/2015/23010/An Aquatic Exercise Program for an Adolescent_With.2.aspx

12. Walton JN, Gardner-Medwin D. Disorders of voluntary muscle. Edinburgh: Waltonn Churchill Livingstone; 1974. 
13. Angelini C, Fanin M. Limb girdle muscular dystrophies: clinicalgenetical diagnostic update and prospects for therapy. Expert Opin Orphan Drugs. 2017;5(10):769-84. https://doi.org/10.1080/21 $\underline{678707.2017 .1367283}$

14. Araujo APQC, Nardes F, Fortes CPDD, Pereira JA, Rebel MF, Dias CM, et al. Consenso brasileiro para distrofia muscular de Duchenne. Parte 2: reabilitação e cuidados sistêmicos. Arq Neuro-Psiquiatr. 2018;76(7):481-9. https://doi.org/10.1590/0004$\underline{282 \times 20180062}$

15. Iwabe C, Miranda-Pfeilsticker B, Nucci A. Medida da função motora: versão da escala para o português e estudo de confiabilidade. Braz J Phys Ther. 2008;12(5):417-24. https://doi. org/10.1590/S1413-35552008000500012

16. Peric M, Peric S, Stevanovic J, Milovanovic S, Basta I, Nikolic A, et al. Quality of life in adult patients with limb-girdle muscular dystrophies. Acta Neurol Belg. 2018;118(2):243-50. https://doi. org/10.1007/s13760-017-0857-9

17. Jacques MF, Stockley R, Onambele-Pearson GL, Reeves ND, Stebbings GK, Dawson EA, et al. Quality of life in adults with muscular dystrophy. Health Qual Life Outcomes. 2019;17(1):121. https://doi.org/10.1186/s12955-019-1177-y

18. Moore $U$, Jacobs $M$, James MK, Mayhew AG, Fernandez-Torron R, Feng J, et al. Assessment of disease progression in dysferlinopathy: A 1-year cohort study. Neurology. 2019;92(5):e461-74. https://doi.org/10.1212/ wnl.0000000000006858
19. Fess EE, Moran CA. American Society of Hand Therapists Clinical Assessment Recommendations [Internet]. American Society of Hand Therapists; 1981. Disponível em: https://n. neurology.org/content/92/5/e461

20. Voet NB, van der Kooi EL, van Engelen BG, Geurts AC. Strength training and aerobic exercise training for muscle disease.

Cochrane Database Syst Rev [Internet]. 2019;12:CD003907.

Disponível em: https://www.cochrane.org/CD003907/

NEUROMUSC strength-training-or-aerobic-exercise-training-

muscle-disease

21. Siciliano G, Simoncini C, Giannotti S, Zampa V, Angelini C, Ricci G. Muscle exercise in limb girdle muscular dystrophies: pitfall and advantages. Acta Myol. 2015;34(1):3-8. Citado em: PMID: $\underline{26155063}$

22. Narayanaswami P, Weiss M, Selcen D, David W, Raynor E, Carter $\mathrm{G}$, et al. Evidence-based guideline summary: diagnosis and treatment of limb-girdle and distal dystrophies: report of the guideline development subcommittee of the American Academy of Neurology and the practice issues review panel of the American Association of Neuromuscular \& Electrodiagnostic Medicine. Neurology. 2014;83(16):1453-63. https://doi.org/10.1212/ wnl.0000000000000892

23. Ellapen TJ, Hammill HV, Swanepoel M, Strydom GL. The benefits of hydrotherapy to patients with spinal cord injuries. Afr J Disabil. 2018;7:450. https://dx.doi.org/10.4102\%2Fajod.v7i0.450

24. Angelini C, Peterle E, Gaiani A, Bortolussi L, Borsato C. Dysferlinopathy course and sportive activity: clues for possible treatment. Acta Myol. 2011;30(2):127-32. Citado em: PMID: $\underline{22106716}$ 